\title{
Properties for certain subclasses of meromorphic functions defined by a multiplier transformation
}

Nak Eun Cho' and Min Yoon²

\section{${ }^{\text {*Correspondence: }}$} myoon@pknu.ac.kr ${ }^{2}$ Department of Statistics, Pukyong National University, 45 Yongso-ro, Busan, 608-737, Korea

Full list of author information is available at the end of the article

\begin{abstract}
Some inclusion and convolution properties of certain subclasses of meromorphic functions associated with a family of multiplier transformations, which are defined by means of the Hadamard product (or convolution), are investigated. We also obtain closure properties for certain integral operators.

MSC: $30 C 45 ; 30 C 80$
\end{abstract}

Keywords: meromorphic function; subordination; starlike of order $\alpha$; convex of order $\alpha$; prestarlike of order $\alpha$; convolution; integral operator

\section{Introduction}

Let $\mathcal{A}$ denote the class of analytic functions $f$ in the open unit disk $\mathbb{U}=\{z \in \mathbb{C}:|z|<1\}$ with the usual normalization $f(0)=f^{\prime}(0)-1=0$. Let $\mathcal{S}^{\prime \prime}(\alpha)$ and $\mathcal{K}(\alpha)$ denote the subclasses of $\mathcal{A}$ consisting of starlike and convex functions of order $\alpha(0 \leq \alpha<1)$ and let $\mathcal{S}^{\prime \prime}(0)=\mathcal{S}^{*}$ and $\mathcal{K}(0)=\mathcal{K}$. If $f$ and $g$ are analytic in $\mathbb{U}$, we say that $f$ is subordinate to $g$ in $\mathbb{U}$, written as $f \prec g$ or $f(z) \prec g(z)$, if there exists a Schwarz function $w$ such that $f(z)=g(w(z))(z \in \mathbb{U})$.

A function $f \in \mathcal{A}$ is said to be prestarlike of order $\alpha$ in $\mathbb{U}$ if

$$
\frac{z}{(1-z)^{2(1-\alpha)}} * f(z) \in \mathcal{S}^{*}(\alpha) \quad(0 \leq \alpha<1)
$$

where $f * g$ denotes the familiar Hadamard product (or convolution) of two analytic functions $f$ and $g$ in $\mathbb{U}$. We denote this class by $\mathcal{R}(\alpha)$ (see, for details, [1]). We note that $\mathcal{R}(0)=\mathcal{K}$ and $\mathcal{R}(1 / 2)=\mathcal{S}^{*}(1 / 2)$.

Let $\mathcal{N}$ be the class of all functions $h$ which are analytic and univalent in $\mathbb{U}$ and for which $h(\mathbb{U})$ is convex with $h(0)=1$.

Let $\mathcal{M}$ denote the class of functions of the form

$$
f(z)=\frac{1}{z}+\sum_{k=0}^{\infty} a_{k} z^{k}
$$

which are analytic in the punctured open unit disk $\mathbb{D}=\mathbb{U} \backslash\{0\}$.

\section{Springer}

C) 2013 Cho and Yoon; licensee Springer. This is an Open Access article distributed under the terms of the Creative Commons Attribution License (http://creativecommons.org/licenses/by/2.0), which permits unrestricted use, distribution, and reproduction in any medium, provided the original work is properly cited. 
For any $n \in \mathbb{N}_{0}=\{0,1,2, \ldots\}$, we denote the multiplier transformations $D_{\lambda}^{n}$ of functions $f \in \mathcal{M}$ by

$$
D_{\lambda}^{n} f(z)=\frac{1}{z}+\sum_{k=0}^{\infty}\left(\frac{k+1+l}{\lambda}\right)^{n} a_{k} z^{k} \quad(\lambda>0 ; z \in \mathbb{D}) .
$$

Obviously, we have

$$
D_{\lambda}^{s}\left(D_{\lambda}^{t} f(z)\right)=D_{\lambda}^{s+t} f(z)
$$

for all nonnegative integers $s$ and $t$. The operators $D_{\lambda}^{n}$ and $D_{1}^{n}$ are the multiplier transformations introduced and studied by Sarangi and Uraligaddi [2] and Uralegaddi and Somanatha $[3,4]$, respectively. Analogous to $D_{\lambda}^{n}$, we here define a new multiplier transformation $I_{\lambda, \mu}^{n}$ as follows.

Let $f_{n}(z)=1 / z+\sum_{k=0}^{\infty}((k+1+\lambda) / \lambda)^{n} z^{k}, n \in \mathbb{N}_{0}$, and let $f_{n, \mu}^{\dagger}$ be such that

$$
f_{n}(z) * f_{n, \mu}^{\dagger}(z)=\frac{1}{z}+\sum_{k=0}^{\infty} \frac{(\mu)_{k+1}}{(1)_{k+1}} z^{k} \quad(\mu>0 ; z \in \mathbb{D}),
$$

where $(v)_{k}$ is the Pochhammer symbol (or the shifted factorial) defined (in terms of the gamma function) by

$$
(v)_{k}:=\frac{\Gamma(v+k)}{\Gamma(v)}= \begin{cases}1 & \text { if } k=0 \text { and } v \in \mathbb{C} \backslash\{0\} \\ v(v+1) \cdots(v+k-1) & \text { if } k \in \mathbb{N}:=\{1,2, \ldots\} \text { and } v \in \mathbb{C}\end{cases}
$$

Then

$$
I_{\lambda, \mu}^{n} f(z)=f_{n, \mu}^{\dagger}(z) * f(z)
$$

We note that $I_{1,2}^{0} f(z)=z f^{\prime}(z)+2 f(z)$ and $I_{1,2}^{1} f(z)=f(z)$. It is easily verified from (1.1) that

$$
z\left(I_{\lambda, \mu}^{n+1} f(z)\right)^{\prime}=\lambda I_{\lambda, \mu}^{n} f(z)-(\lambda+1) I_{\lambda, \mu}^{n+1} f(z)
$$

and

$$
z\left(I_{\lambda, \mu}^{n} f(z)\right)^{\prime}=\mu I_{\lambda, \mu+1}^{n} f(z)-(\mu+1) I_{\lambda, \mu}^{n} f(z)
$$

The definition (1.1) of the multiplier transformation $I_{\lambda, \mu}^{n}$ is motivated essentially by the Choi-Saigo-Srivastava operator [5] for analytic functions, which includes the Noor integral operator studied by Liu [6] (also, see [7-9]).

We also define the function $\phi(a, c ; z)$ by

$$
\phi(a, c ; z):=\frac{1}{z}+\sum_{k=0}^{\infty} \frac{(a)_{k+1}}{(c)_{k+1}} z^{k} \quad\left(z \in \mathbb{U} ; a \in \mathbb{R} ; c \in \mathbb{R} \backslash \mathbb{Z}_{0}^{-} ; \mathbb{Z}_{0}^{-}:=\{-1,-2, \ldots\}\right)
$$

By using the operator $I_{\lambda, \mu}^{n}$, we introduce the following class of analytic functions for $\gamma>0, \lambda>0, s \in \mathbb{R}, \mu>0$ and $h \in \mathcal{N}$ :

$$
\mathcal{M}_{\lambda, \mu}^{n}(\gamma ; h):=\left\{f \in \mathcal{M}:(1-\gamma) z I_{\lambda, \mu}^{n} f(z)+\gamma z^{2}\left(I_{\lambda, \mu}^{n} f(z)\right)^{\prime} \prec h(z)\right\}
$$


In the present paper, we derive some inclusion relations, convolution properties and integral preserving properties for the class $\mathcal{M}_{\lambda, \mu}^{n}(\gamma ; h)$.

The following lemmas will be required in our investigation.

Lemma 1.1 [10, Lemma 2, p.192] Let $g$ be analytic in $\mathbb{U}$ and $h$ be analytic and convex univalent in $U$ with $h(0)=g(0)$. If

$$
g(z)+\frac{1}{\gamma} z g^{\prime}(z) \prec h(z) \quad(\operatorname{Re}\{\gamma\} \geq 0 ; \gamma \neq 0)
$$

then

$$
g(z) \prec \widetilde{h}(z)=\gamma z^{-\gamma} \int_{0}^{z} t^{\gamma-1} h(t) d t \prec h(z)
$$

and $\tilde{h}$ is the best dominant of (1.5).

Lemma 1.2 [1, Theorem 2.4, p.54] Let $f \in \mathcal{S}^{\prime \prime}(\alpha)$ and $g \in \mathcal{R}(\alpha)$. Then for any analytic function $F$ in $\mathbb{U}$,

$$
\frac{g *(f F)}{g * f}(\mathbb{U}) \subset \overline{c o}(F(\mathbb{U})),
$$

where $\overline{c o}(F(\mathbb{U}))$ denotes the convex hull of $F(\mathbb{U})$.

Lemma 1.3 [11, Lemma 5, p.656] Let $0<a \leq c$. Then

$$
\operatorname{Re}\{z \phi(a, c ; z)\}>\frac{1}{2} \quad(z \in \mathbb{U})
$$

where $\phi$ is given by (1.4).

\section{Inclusion relations}

Theorem 2.1 If $0 \leq \gamma_{1}<\gamma_{2}$, then

$$
\mathcal{M}_{\lambda, \mu}^{n}\left(\gamma_{2} ; h\right) \subset \mathcal{M}_{\lambda, \mu}^{n}\left(\gamma_{1} ; h\right) .
$$

Proof Let

$$
g(z)=z I_{\lambda, \mu}^{n} f(z) \quad\left(f \in \mathcal{M}_{\lambda, \mu}^{n}\left(\gamma_{2} ; h\right): z \in \mathbb{U}\right) .
$$

Then the function $g$ is analytic in $\mathbb{U}$ with $g(0)=1$. Differentiating both sides of (2.1), we have

$$
\left(1+\gamma_{2}\right) z I_{\lambda, \mu}^{n} f(z)+\gamma_{2} z^{2}\left(I_{\lambda, \mu}^{n} f(z)\right)^{\prime}=g(z)+\gamma_{2} z g^{\prime}(z) \prec h(z) .
$$

Hence an application of Lemma 1.1 with $\mu=1 / \gamma_{2}$ yields

$$
g(z) \prec h(z) .
$$


Since $0 \leq \gamma_{1} / \gamma_{2}<1$ and $h$ is convex univalent in $U$, it follows from (2.1), (2.2) and (2.3) that

$$
\begin{aligned}
(1 & \left.+\gamma_{1}\right) z I_{\lambda, \mu}^{n} f(z)+\gamma_{1} z^{2}\left(I_{\lambda, \mu}^{n} f(z)\right)^{\prime} \\
& =\frac{\gamma_{1}}{\gamma_{2}}\left[\left(1-\gamma_{2}\right) z I_{\lambda, \mu}^{n} f(z)+\gamma_{2} z^{2}\left(I_{\lambda, \mu}^{n} f(z)\right)^{\prime}\right]+\left(1-\frac{\gamma_{1}}{\gamma_{2}}\right) g(z) \\
& \prec h(z) .
\end{aligned}
$$

Therefore $f \in \mathcal{M}_{\lambda, \mu}^{n}\left(\gamma_{1} ; h\right)$, and so we complete the proof of Theorem 2.1.

Theorem 2.2 If $0<\mu_{1} \leq \mu_{2}$, then

$$
\mathcal{M}_{\lambda, \mu_{2}}^{n}(\gamma ; h) \subset \mathcal{M}_{\lambda, \mu_{1}}^{n}(\gamma ; h)
$$

Proof Let $f \in \mathcal{M}_{\lambda, \mu_{2}}^{n}(\gamma ; h)$. Then

$$
\begin{aligned}
(1 & +\gamma) z I_{\lambda, \mu_{1}}^{n} f(z)+\gamma z^{2}\left(I_{\lambda, \mu_{1}}^{n} f(z)\right)^{\prime} \\
& =z \phi\left(\mu_{1}, \mu_{2} ; z\right) *\left[(1+\gamma) z I_{\lambda, \mu_{2}}^{n} f(z)+\gamma z^{2}\left(I_{\lambda, \mu_{2}}^{n} f(z)\right)^{\prime}\right] .
\end{aligned}
$$

In view of Lemma 1.3, we see that the function $z \phi\left(\mu_{1}, \mu_{2} ; z\right)$ has the Herglotz representation

$$
z \phi\left(\mu_{1}, \mu_{2} ; z\right)=\int_{|x|=1} \frac{d \mu(x)}{1-x z} \quad(z \in \mathbb{U})
$$

where $\mu(x)$ is a probability measure defined on the unit circle $|x|<1$ and

$$
\int_{|x|=1} d \mu(x)=1
$$

Since $h$ is convex univalent in $\mathbb{U}$, it follows from (2.4) and (2.5) that

$$
(1+\gamma) z I_{\lambda, \mu_{1}}^{n} f(z)+\gamma z^{2}\left(I_{\lambda, \mu 1}^{n} f(z)\right)^{\prime}=\int_{|x|=1} h(x z) d \mu(x) \prec h(z),
$$

which completes the proof of Theorem 2.2.

Theorem 2.3 If $\mu>0$, then

$$
\mathcal{M}_{\lambda, \mu+1}^{n}(\gamma ; \tilde{h}) \subset \mathcal{M}_{\lambda, \mu}^{n}(\gamma ; h)
$$

where

$$
\widetilde{h}(z)=\mu z^{-\mu} \int_{0}^{z} t^{\mu-1} h(t) d t \prec h(z) .
$$

Proof Let

$$
g(z)=(1+\gamma) z I_{\lambda, \mu}^{n} f(z)+\gamma z^{2}\left(I_{\lambda, \mu}^{n} f(z)\right)^{\prime} \quad(f \in \mathcal{M} ; z \in \mathbb{U}) .
$$


Then from (1.4) and (2.6), we have

$$
z^{-1} g(z)=\gamma \mu I_{\lambda, \mu+1}^{n} f(z)+(1-\gamma \mu) I_{\lambda, \mu}^{n} f(z) .
$$

Differentiating both sides of (2.6) and using (1.4), we obtain

$$
\begin{aligned}
& z^{-1}\left(z g^{\prime}(z)+g(z)\right) \\
& \quad=\gamma \mu z\left(I_{\lambda, \mu+1}^{n} f(z)\right)+(1-\gamma \mu)\left(\mu I_{\lambda, \mu+1}^{n} f(z)-(\mu+1) I_{\lambda, \mu}^{n} f(z)\right) .
\end{aligned}
$$

By a simple calculation with (2.7) and (2.8), we get

$$
g(z)+\frac{z g^{\prime}(z)}{\mu}=(1+\gamma) \frac{I_{\lambda, \mu+1}^{n} f(z)}{z}+\gamma\left(I_{\lambda, \mu+1}^{n} f(z)\right)^{\prime}
$$

If $f \in \mathcal{M}_{\lambda, \mu+1}^{n}(\gamma ; h)$, then it follows from (2.9) that

$$
g(z)+\frac{z g^{\prime}(z)}{\mu} \prec h(z) \quad(\mu>0) .
$$

Hence an application of Lemma 1.1 yields

$$
g(z) \prec \widetilde{h}(z)=\mu z^{-\mu} \int_{0}^{z} t^{\mu-1} h(t) d t \prec h(z),
$$

which shows that

$$
f \in \mathcal{M}_{\lambda, \mu+1}^{n}(\gamma ; \tilde{h}) \subset \mathcal{M}_{\lambda, \mu}^{n}(\gamma ; h)
$$

Theorem 2.4 If $s \in \mathbb{R}$ and $\lambda>0$, then

$$
\mathcal{M}_{\lambda, \mu}^{n}(\gamma ; \tilde{h}) \subset \mathcal{M}_{\lambda, \mu}^{n+1}(\gamma ; h)
$$

where

$$
\widetilde{h}(z)=\lambda z^{-\lambda} \int_{0}^{z} t^{\lambda-1} h(t) d t \prec h(z) .
$$

Proof By using the same techniques as in the proof of Theorem 2.3 and (1.5), we have Theorem 2.4 and so we omit the detailed proof involved.

Theorem 2.5 Let $\gamma>0, \beta>0$ and $f \in \mathcal{M}_{\lambda, \mu}^{n}(\gamma ; \beta h+1-\beta)$. If $\beta \leq \beta_{0}$, where

$$
\beta_{0}=\frac{1}{2}\left(1-\frac{1}{\gamma} \int_{0}^{1} \frac{u^{\frac{1}{\gamma}-1}}{1+u} d u\right)^{-1}
$$

then $f \in \mathcal{M}_{\lambda, \mu}^{n}(0 ; h)$. The bound $\beta_{0}$ is sharp for the function

$$
h(z)=\frac{1}{1-z} \quad(z \in \mathbb{U})
$$


Proof Let

$$
g(z)=z I_{\lambda, \mu}^{n} f(z) \quad\left(f \in \mathcal{M}_{\lambda, \mu}^{n}(\gamma ; \beta h+1-\beta) ; \gamma>0 ; \beta>0\right) .
$$

Then we have

$$
\begin{aligned}
g(z)+\gamma z g^{\prime}(z) & =(1+\gamma) z I_{\lambda, \mu}^{n} f(z)+\gamma z^{2}\left(I_{\lambda, \mu}^{n} f(z)\right)^{\prime} \\
& \prec \beta h(z)+1-\beta .
\end{aligned}
$$

Hence an application of Lemma 1.1 yields

$$
g(z) \prec \frac{\beta}{\gamma} z^{-\frac{1}{\gamma}} \int_{0}^{z} t^{\frac{1}{\gamma}-1} h(t) d t+1-\beta=(h * \psi)(z),
$$

where

$$
\psi(z)=\frac{\beta}{\gamma} z^{-\frac{1}{\gamma}} \int_{0}^{z} \frac{t^{\frac{1}{\gamma}-1}}{1-t} d t+1-\beta .
$$

If $0<\beta \leq \beta_{0}$, where $\beta_{0}$ is given by (2.10), then from (2.13), we have

$$
\begin{aligned}
\operatorname{Re}\{\psi(z)\} & =\frac{\beta}{\gamma} \int_{0}^{1} u^{\frac{1}{\gamma}-1} \operatorname{Re}\left\{\frac{1}{1-u z} d u\right\}+1-\beta \\
& >\frac{\beta}{\gamma} \int_{0}^{1} \frac{u^{\frac{1}{\gamma}-1}}{1+u} d u+1-\beta \\
& \geq \frac{1}{2} .
\end{aligned}
$$

By using the Herglotz representation for $\psi$, it follows from (2.11) and (2.12) that

$$
z I_{\lambda, \mu}^{n} f(z) \prec(h * \psi)(z) \prec h(z)
$$

since $h$ is convex univalent in $\mathbb{U}$. This shows that $f \in \mathcal{M}_{\lambda, \mu}^{n}(0 ; h)$.

For $h(z)=1 /(1-z)$ and $f \in \mathcal{M}$ defined by

$$
z I_{\lambda, \mu}^{n} f(z)=\frac{\beta}{\gamma} z^{-\frac{1}{\gamma}} \int_{0}^{z} \frac{t^{\frac{1}{\gamma}-1}}{1-t} d t+1-\beta
$$

it is easy to verify that

$$
(1+\gamma) z I_{\lambda, \mu}^{n} f(z)+\gamma z^{2}\left(I_{\lambda, \mu}^{n} f(z)\right)^{\prime}=\beta h(z)+1-\beta .
$$

Thus $f \in \mathcal{M}_{\lambda, \mu}^{n}(\gamma ; \beta h+1-\beta)$. Furthermore, for $\beta>\beta_{0}$, we have

$$
\operatorname{Re}\left\{z I_{\lambda, \mu}^{n} f(z)\right\} \rightarrow \frac{\beta}{\gamma} \int_{0}^{1} \frac{u^{\frac{1}{\gamma}-1}}{1+u} d u+1-\beta<\frac{1}{2} \quad(z \rightarrow-1),
$$

which implies that $f \notin \mathcal{M}_{\lambda, \mu}^{n}(0 ; h)$. Hence the bound $\beta_{0}$ cannot be increased when $h(z)=$ $1 /(1-z)(z \in \mathbb{U})$. 


\section{Convolution properties}

Theorem 3.1 Iff $\in \mathcal{M}_{\lambda, \mu}^{n}(\gamma ; h)$ and

$$
\operatorname{Re}\{z g(z)\}>\frac{1}{2} \quad(g \in \mathcal{M} ; z \in \mathbb{U})
$$

then

$$
f * g \in \mathcal{M}_{\lambda, \mu}^{n}(\gamma ; h)
$$

Proof Let $f \in \mathcal{M}_{\lambda, \mu}^{n}(\gamma ; h)$ and $g \in \mathcal{M}$. Then we have

$$
(1+\gamma) z I_{\lambda, \mu}^{n}(f * g)(z)+\gamma z^{2}\left(I_{\lambda, \mu}^{n}(f * g)(z)\right)^{\prime}=z g(z) * \psi(z)
$$

where

$$
\psi(z)=(1+\gamma) z \frac{I_{\lambda, \mu}^{n} f(z)}{+} \gamma z^{2}\left(I_{\lambda, \mu}^{n} f(z)\right)^{\prime} \prec h(z) .
$$

The remaining part of the proof of Theorem 3.1 is similar to that of Theorem 2.2, and so we omit the details involved.

Corollary 3.1 Let $f \in \mathcal{M}_{\lambda, \mu}^{n}(\gamma ; h)$ be given by (1.1). Then the function

$$
\sigma_{m}(z)=\int_{0}^{1} t S_{m}(t z) d t \quad(z \in \mathbb{U})
$$

where

$$
S_{m}(z)=\frac{1}{z}+\sum_{n=1}^{m-1} a_{n} z^{n-1} \quad(m \in \mathbb{N} \backslash\{1\} ; z \in \mathbb{U}),
$$

is also in the class $\mathcal{M}_{\lambda, \mu}^{n}(\gamma ; h)$.

Proof We have

$$
\sigma_{m}(z)=\frac{1}{z}+\sum_{n=1}^{m-1} \frac{a_{n}}{n-1} z^{n+1}=\left(f * g_{m}\right)(z) \quad(m \in \mathbb{N} \backslash\{1\}),
$$

where

$$
f(z)=\frac{1}{z}+\sum_{n=1}^{\infty} a_{n} z^{n-1} \in \mathcal{M}_{\lambda, \mu}^{n}(\gamma ; h)
$$

and

$$
g_{m}(z)=\frac{1}{z}+\sum_{n=1}^{m-1} \frac{z^{n}}{n-1} \in \mathcal{M},
$$


while, it is known [4] that

$$
\operatorname{Re}\left\{z g_{m}(z)\right\}=\operatorname{Re}\left\{1+\sum_{n=1}^{m-1} \frac{z^{n}}{n+1}\right\}>\frac{1}{2} \quad(m \in \mathbb{N} \backslash\{1\} ; z \in \mathbb{U}) .
$$

In view of (3.1) and (3.2), an application of Theorem 3.1 leads to $\sigma_{m} \in \mathcal{M}_{\lambda, \mu}^{n}(\gamma ; h)$.

Theorem 3.2 Iff $\in \mathcal{M}_{\lambda, \mu}^{n}(\gamma ; h)$ and

$$
z^{2} g(z) \in R(\alpha) \quad(g \in \mathcal{M} ; z \in \mathbb{U})
$$

then

$$
(f * g) \in \mathcal{M}_{\lambda, \mu}^{n}(\gamma ; h)
$$

Proof By using a similar method as in the proof of Theorem 3.1, we have

$$
(1+\gamma) z I_{\lambda, \mu}^{n}(f * g)(z)+\gamma z^{2}\left(I_{\lambda, \mu}^{n}(f * g)(z)\right)^{\prime}=\frac{z^{2} g(z) *(z \psi(z))}{z^{2} g(z) * z} \quad(z \in \mathbb{U}),
$$

where

$$
\psi(z)=(1+\gamma) z I_{\lambda, \mu}^{n} f(z)+\gamma z^{2}\left(I_{\lambda, \mu}^{n} f(z)\right)^{\prime} \prec h(z) .
$$

Since $h$ is convex univalent in $\mathbb{U}$, it follows from (3.3) and Lemma 1.2 that Theorem 3.2 holds true.

If we take $\alpha=0$ and $\alpha=1 / 2$ in Theorem 3.2, we have the following corollary.

Corollary 3.2 If $\in \mathcal{M}_{\lambda, \mu}^{n}(\gamma ; h)$ and $g \in \mathcal{M}$ satisfies one of the following conditions:

(i) $z^{2} g(z)$ is convex univalent in $\mathbb{U}$

or

(ii) $z^{2} g(z) \in S^{*}\left(\frac{1}{2}\right)$,

then $(f * g) \in \mathcal{M}_{\lambda, \mu}^{n}(\gamma ; h)$.

\section{Integral operators}

Theorem 4.1 Iff $\in \mathcal{M}_{\lambda, \mu}^{n}(\gamma ; h)$, then the function $F$ defined by

$$
F(z)=\frac{c-1}{z^{c}} \int_{0}^{z} t^{c-1} f(t) d t \quad(\operatorname{Re}\{c\}>1)
$$

is in the class $\mathcal{M}_{\lambda, \mu}^{n}(\gamma ; \widetilde{h})$, where

$$
\widetilde{h}(z)=(c-1) z^{-(c-1)} \int_{0}^{z} t^{c} h(t) d t \prec h(z) .
$$

Proof Let $f \in \mathcal{M}_{\lambda, \mu}^{n}(\gamma ; h)$. Then from (4.1), we obtain

$$
(c-1) f(z)=z F^{\prime}(z)+c F(z) .
$$


Define the function $G$ by

$$
z^{-1} G(z)=(1+\gamma) I_{\lambda, \mu}^{n} F(z)+\gamma z\left(I_{\lambda, \mu}^{n} F(z)\right)^{\prime} \quad(z \in \mathbb{D}) .
$$

Differentiating both sides of (4.3) with respect to $z$, we get

$$
z G^{\prime}(z)-G(z)=(1+\gamma) z I_{\lambda, \mu}^{n}\left(z F^{\prime}(z)\right)+\gamma z^{2}\left(I_{\lambda, \mu}^{n}\left(z F^{\prime}(z)\right)\right)^{\prime} .
$$

Furthermore, it follows from (4.2), (4.3) and (4.4) that

$$
\begin{aligned}
(1+\gamma) z I_{\lambda, \mu}^{n} f(z)+\gamma z^{2}\left(I_{\lambda, \mu}^{n} f(z)\right)^{\prime} \\
=(1+\gamma) z I_{\lambda, \mu}^{n}\left(\frac{z F^{\prime}(z)+c F(z)}{c-1}\right) \\
\quad+\gamma z^{2}\left(I_{\lambda, \mu}^{n}\left(\frac{z F^{\prime}(z)+c F(z)}{c-1}\right)\right)^{\prime} \\
=\frac{c}{c-1} G(z)+\frac{1}{c-1}\left(z G^{\prime}(z)-G(z)\right) \\
=G(z)+\frac{1}{c-1} z G^{\prime}(z) .
\end{aligned}
$$

Since $f \in \mathcal{M}_{\lambda, \mu}^{n}(\gamma ; h)$, from (4.5), we have

$$
G(z)+\frac{1}{c-1} z G^{\prime}(z) \prec h(z) \quad(\operatorname{Re}\{c\}>1),
$$

and so an application of Lemma 1.1 yields

$$
G(z) \prec \widetilde{h}(z)=\frac{c-1}{z^{c-1}} \int_{0}^{z} t^{c} h(t) d t \prec h(z) .
$$

Therefore we conclude that

$$
F \in \mathcal{M}_{\lambda, \mu}^{n}(\gamma ; \widetilde{h}) \subset \mathcal{M}_{\lambda, \mu}^{n}(\gamma ; h)
$$

Theorem 4.2 Iff $\in \mathcal{M}$ and $F$ are defined as in Theorem 4.1, if

$$
(1-\alpha) z I_{\lambda, \mu}^{n} F(z)+\alpha z I_{\lambda, \mu}^{n} f(z) \prec h(z) \quad(\alpha>0),
$$

then $F \in \mathcal{M}_{\lambda, \mu}^{n}(0 ; \widetilde{h})$, where

$$
\widetilde{h}(z)=\frac{c-1}{\alpha} z^{-\frac{\alpha}{c-1}} \int_{0}^{z} t^{\frac{c-1}{\alpha}-1} h(t) \prec h(z) \quad(\operatorname{Re}\{c\}>1) .
$$

Proof Let

$$
G(z)=z I_{\lambda, \mu}^{n} F(z) \quad(z \in \mathbb{D}) .
$$

Then $G$ is analytic in $\mathbb{U}$ with $G(0)=1$ and

$$
z G^{\prime}(z)=z^{2}\left(I_{\lambda, \mu}^{n} F(z)\right)^{\prime}+G(z) .
$$


It follows from (4.2), (4.6), (4.7) and (4.8) that

$$
\begin{aligned}
(1 & -\alpha) z I_{\lambda, \mu}^{n} F(z)+\alpha z I_{\lambda, \mu}^{n} f(z) \\
& =(1-\alpha) z \frac{I_{\lambda, \mu}^{n} F(z)}{+} \frac{\alpha}{c-1}\left[c z I_{\lambda, \mu}^{n} F(z)+z^{2}\left(I_{\lambda, \mu}^{n} F(z)\right)^{\prime}\right] \\
& =G(z)+\frac{\alpha}{c-1} z G^{\prime}(z) \prec h(z) \quad(\operatorname{Re}\{c\}>1 ; \alpha>0) .
\end{aligned}
$$

Therefore, by Lemma 1.1, we conclude that Theorem 4.2 holds true as stated.

Theorem 4.3 Let $F \in \mathcal{M}_{\lambda, \mu}^{n}(\gamma ; h)$. If the function $f$ is defined by

$$
F(z)=\frac{c-1}{z^{c}} \int_{0}^{z} t^{c-1} f(t) d t \quad(c>1)
$$

then

$$
\sigma f(\sigma z) \in \mathcal{M}_{\lambda, \mu}^{n}(\gamma ; h)
$$

where

$$
\sigma=\sigma(c)=\frac{\sqrt{1+(c-1)^{2}}-1}{c-1} .
$$

The bound $\sigma$ is sharp for the function

$$
h(z)=\beta+(1-\beta) \frac{1+z}{1-z} \quad(\beta \neq 1 ; z \in \mathbb{U}) .
$$

Proof We note that for $F \in \mathcal{M}$,

$$
F(z)=F(z) * \frac{1}{z(1-z)} \quad \text { and } \quad z F^{\prime}(z)=F(z) *\left(\frac{1}{z(1-z)^{2}}-\frac{2}{z^{2}(1-z)}\right) .
$$

Then from (4.9), we have

$$
f(z)=\frac{c F(z)+z F^{\prime}(z)}{c-1}=(F * g)(z) \quad(c>1 ; z \in \mathbb{D}),
$$

where

$$
g(z)=\frac{1}{c-1}\left((c-2) \frac{1}{z(1-z)}+\frac{1}{z(1-z)^{2}}\right) \in \mathcal{M} .
$$

Next, we show that

$$
\operatorname{Re}\{z g(z)\}>\frac{1}{2} \quad(|z|<\sigma)
$$

where $\sigma=\sigma(c)$ is given by (4.10). Letting

$$
\frac{1}{1-z}=\operatorname{Re}^{i \theta} \quad(|z|=r<1 ; R>0),
$$


we see that

$$
\cos \theta=\frac{1+R^{2}\left(1-r^{2}\right)}{2 R} \quad \text { and } \quad R \geq \frac{1}{1+r} .
$$

Then for (4.13) and (4.15), we have

$$
\begin{aligned}
2 \operatorname{Re}\{z g(z)\} & =\frac{2}{c-1}\left[(c-2) R \cos \theta+R^{2}\left(2 \cos ^{2} \theta-1\right)\right] \\
& =\frac{R^{2}}{c-1}\left[c\left(1-r^{2}\right)+R^{2}\left(1-r^{2}\right)^{2}-2\right]+1 \\
& \geq \frac{R^{2}}{c-1}\left[c-1-2 r-(c-1) r^{2}\right]+1 .
\end{aligned}
$$

This evidently gives (4.14), which is equivalent to

$$
\operatorname{Re}\{\sigma z g(\sigma z)\}>\frac{1}{2} \quad(z \in \mathbb{U}) .
$$

Let $F \in \mathcal{M}_{\lambda, \mu}^{n}(\gamma ; h)$. Then, by using (4.12) and (4.16), an application of Theorem 3.1 yields

$$
\sigma f(\sigma z)=F(z) * \sigma g(\sigma z) \in \mathcal{M}_{\lambda, \mu}^{n}(\gamma ; h) .
$$

For $h$ given by (4.11), we consider the function $F \in \mathcal{M}$ defined by

$$
(1+\gamma) z I_{\lambda, \mu}^{n} F(z)+\gamma z^{2}\left(I_{\lambda, \mu}^{n} F(z)\right)^{\prime}=\beta+(1-\beta) \frac{1+z}{1-z} \quad(\beta \neq 1 ; z \in \mathbb{U}) .
$$

Then from (4.3), (4.5) and (4.17), we find that

$$
\begin{aligned}
(1+\gamma) z I_{\lambda, \mu}^{n} f(z)+\gamma z^{2}\left(I_{\lambda, \mu}^{n} f(z)\right)^{\prime} \\
=\beta+(1-\beta) \frac{1+z}{1-z}+\frac{z}{c-1}\left(\beta+(1-\beta) \frac{1+z}{1-z}\right)^{\prime} \\
=\beta+\frac{(1-\beta)\left(c-1+2 z-(c-1) z^{2}\right)}{(c-1)(1-z)^{2}} \\
=\beta \quad(z=-\sigma) .
\end{aligned}
$$

Therefore we conclude that the bound $\sigma=\sigma(c)$ cannot be increased for each $c(c>1)$.

\section{Competing interests}

The authors declare that they have no competing interests.

\section{Authors' contributions}

All authors jointly worked on the results and they read and approved the final manuscript.

\section{Author details}

${ }^{1}$ Department of Applied Mathematics, Pukyong National University, 45 Yongso-ro, Busan, 608-737, Korea. ${ }^{2}$ Department of Statistics, Pukyong National University, 45 Yongso-ro, Busan, 608-737, Korea.

\section{Acknowledgements}

The authors would like to express their thanks to the referees for valuable advice regarding a previous version of this paper. This research was supported by the Basic Science Research Program through the National Research Foundation of Korea (NRF) funded by the Ministry of Education, Science and Technology (No. 2012-0002619). 


\section{References}

1. Ruscheweyh, S: Convolution in Geometric Function Theory. Presses University Montreal, Montreal (1982)

2. Sarangi, SM, Uralegaddi, SB: Certain differential operators for meromorphic functions. Bull. Calcutta Math. Soc. 88 333-336 (1996)

3. Uralegaddi, BA, Somanatha, C: New criteria for meromorphic starlike functions. Bull. Aust. Math. Soc. 43, 137-140 (1991)

4. Uralegaddi, BA, Somanatha, C: Certain differential operators for meromorphic functions. Houst. J. Math. 17, 279-284 (1991)

5. Choi, JH, Sagio, M, Srivastava, HM: Some inclusion properties of a certain family of integral operators. J. Math. Anal. Appl. 276, 432-445 (2002)

6. Liu, J-L: The Noor integral and strongly starlike functions. J. Math. Anal. Appl. 261, 441-447 (2001)

7. Liu, J-L, Noor, Kl: Some properties of Noor integral operator. J. Nat. Geom. 21, 81-90 (2002)

8. Noor, Kl: On new classes of integral operators. J. Nat. Geom. 16, 71-80 (1999)

9. Noor, KI, Noor, MA: On integral operators. J. Math. Anal. Appl. 238, 341-352 (1999)

10. Hallenbeck, DJ, Ruscheweyh, S: Subordination by convex functions. Proc. Am. Math. Soc. 52, 191-195 (1975)

11. Lucyna, T-S: On certain applications of the Hadamard product. Appl. Math. Comput. 199, 653-662 (2008)

doi:10.1186/1029-242X-2013-46

Cite this article as: Cho and Yoon: Properties for certain subclasses of meromorphic functions defined by a multiplier transformation. Journal of Inequalities and Applications 2013 2013:46.

\section{Submit your manuscript to a SpringerOpen ${ }^{\ominus}$ journal and benefit from:}

- Convenient online submission

- Rigorous peer review

- Immediate publication on acceptance

- Open access: articles freely available online

- High visibility within the field

- Retaining the copyright to your article 